\title{
Inkjet Printed GPS Antenna on a 3D Printed Substrate using Low-Cost Machines
}

\author{
J. Heirons ${ }^{1}$, S. Jun ${ }^{1}$, A. Shastri ${ }^{1}$, B. Sanz-Izquierdo ${ }^{1}$, David Bird ${ }^{2}$, L. Winchester ${ }^{2}$, L.Evans ${ }^{2}$, and A. McClelland ${ }^{2}$ \\ ${ }^{1}$ School of Engineering and Digital Arts, The University of Kent, CT27NT, Canterbury, Kent, UK \\ b.sanz@kent.ac.uk, sj329@kent.ac.uk. as2024@,kent.ac.uk
${ }^{2}$ The Centre for Process Innovation (CPI), Thomas Wright Way, NETPark, Sedgefield, Co Durham, United Kingdom, TS21 3FG
David.Bird@uk-cpi.com, Alan.McClelland@uk-cpi.com

\begin{abstract}
A circularly polarized patch antenna fabricated using commercially available, low-cost, printers is described. Two additive processes are combined for the fabrication: stereolithography (SLA) and inkjet printing of silver inks. A widely available SLA 3D printer is employed to fabricate the substrate of the antenna. Inkjet printing is used to deposit the metallic layers of the radiating element on the substrate. The two machines employed are very lowcost in comparison to those used in previously reported work. Good adhesion of the metallic patterns to the substrates is observed. Furthermore, the resistance of the added metallic patch is relatively low. The aim is to demonstrate the use of alternative, inexpensive machines, for the prototyping and manufacturing of antennas on 3D printed substrates. In this work, the antenna operates at the 1.575GHz GPS frequency band. Finite-difference-timedomain simulations compare well with the practical experiments.
\end{abstract}

Keywords - 3D printing; Additive Manufacturing, circular polarisation, Patch Antenna

\section{INTRODUCTION}

Additive manufacturing (AM), also known as 3D printing, is a process of fabricating a 3D digital design by printing layer after layer. 3D printing has advanced very rapidly in recent years and has become an alternative to traditional manufacture methods for customized objects. Originally intended for the prototyping of mechanical objects, this technique has expanded into different areas such as biomedical [1] and electronics [2]. Within electronics, antennas and microwave engineering can greatly benefit from this technology. Researchers have already demonstrated the potential applicability of $3 \mathrm{D}$ printing in this field. Light weight waveguides have been fabricated by copper plating plastics forms [3]. Substrates for antenna applications have been modified and new properties have been found with the assistance of additive manufacturing [4]. Novel frequency selective structures (FSS) have been developed by fully [5] and partially [6] metalizing 3D printed elements. Non-uniform electromagnetic band gap structures have been fabricated on printed substrates [7]. Antennas have been placed onto wearables and tested on 3D printed phantoms [8-9].

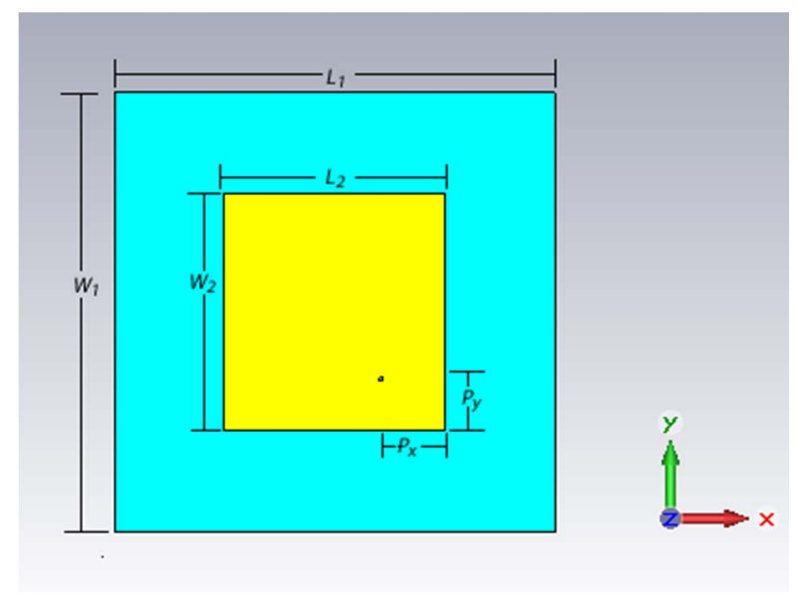

Fig.1. Antenna design with dimensions

Another area were additive manufacturing has advance rapidly is in the development of nanoparticle silver inks that can be used for inkjet printing technology. This has led to many papers on electronic devices using this technique. Most of the published work have used planar, uniform and thin sheets made of cellulose or polyethylene terephthalate (PET). Sensors [10], RFID Tags [11]-[12], RF energy harvesting devices [13], antennas [14] and FSS [15] have been reported. An antenna for energy harvesting applications has recently been proposed were inkjet printing of nanosilver inks is combined with 3D printed substrates [16]. The work was carried out using printing equipment which is relatively expensive and inaccessible for home use.

This paper presents the use of low cost printers for the development of antennas which are entirely fabricated by additive processes. Inkjet printing of silver conductive ink is combined with stereolithography (SLA) printing to achieve this. The design used for this demonstration is a circularly polarized (CP) patch antenna, designed to work on the GPS L1 frequency band. The finite-difference time-domain method included in CST Microwave Studio ${ }^{\mathrm{TM}}$ was used for all the simulations in this paper. 
TABLE I.

\section{ANTENNA DESIGN AND SimUlations}

An illustration of the circularly polarized patch antenna is shown in Fig. 1. The main dimensions are given in Table I. The antenna consists of a rectangular patch element on a square substrate of side $100 \mathrm{~mm}$ and height $3 \mathrm{~mm}$. In order to obtain the required right hand circular polarization ( $\mathrm{RHCP})$, the antenna is probe fed with the location of the probe placed on the diagonal from the top left corner to the bottom right corner. The dielectric permittivity of SLA printed substrates were measured externally through a transmission line system, and was given as 2.83 with loss tangent of 0.038 .

The simulated reflection coefficient of the antenna is shown in Fig. 2. The designed antenna had a resonant frequency at $1.575 \mathrm{MHz}$ and a $-10 \mathrm{~dB}$ bandwidth of $7 \%$. The simulated axial ratio seen in Fig. 3 indicates circular polarization at the intended frequency band.

\section{FABRICATION AND MEASUREMENTS}

\section{A. Fabrication}

The Formlabs Form 1+ printer [17] was used for the fabrication of the substrate. The Form 1+ uses a stereolithography (SLA) process where a laser cures layers of UV sensitive resin. The process gives a smoother finish, compared to the alternative low-cost 3D printer methods. This makes it more suitable for combining with inkjet printing as it gives a better surface to bond the silver conductive ink onto. The resin selected was the clear material provided by Formlabs [17]. A $100 \times 100 \times 3 \mathrm{~mm}$ substrate was printed using the SLA machine. 30 layers of thickness $0.1 \mathrm{~mm}$ were necessary to achieve the required height. The surface roughness was measured to be $206.2 \mathrm{~nm}$ and the $2 \mathrm{D}$ representation of the surface profile can be seen in Fig. 4.

The inkjet printer used was the Argentum from Cartesian Co. [18]. The printer is designed specifically for printing silver conductive ink. It uses two cartridges in tandem to print each layer, printing a layer of silver nitrate solution and then a layer of ascorbic acid (Vitamin C) which chemically reacts to leave the silver metalized layer. Any excess is washed off as the solution is water soluble. An investigation of this chemical processes indicating the conductivities achieved on various substrates is reported in [19]. As expected, it was found that the greater the number of layers the lower the resistance.
ANTENNA DIMENSIONS

\begin{tabular}{|c|c|c|c|c|c|c|}
\hline & $W_{1}$ & $L_{1}$ & $W_{2}$ & $L_{2}$ & $P_{x}$ & $P_{y}$ \\
\hline $\begin{array}{c}\text { Dimensions } \\
(\mathrm{mm})\end{array}$ & 100 & 100 & 53.6 & 50.4 & 14.6 & 11.6 \\
\hline
\end{tabular}

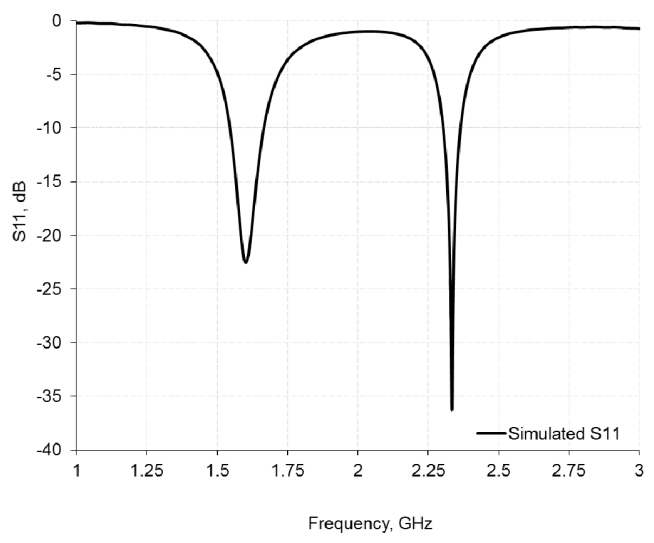

Fig.2 Simulated reflection coefficient $\left(\mathrm{S}_{11}\right)$

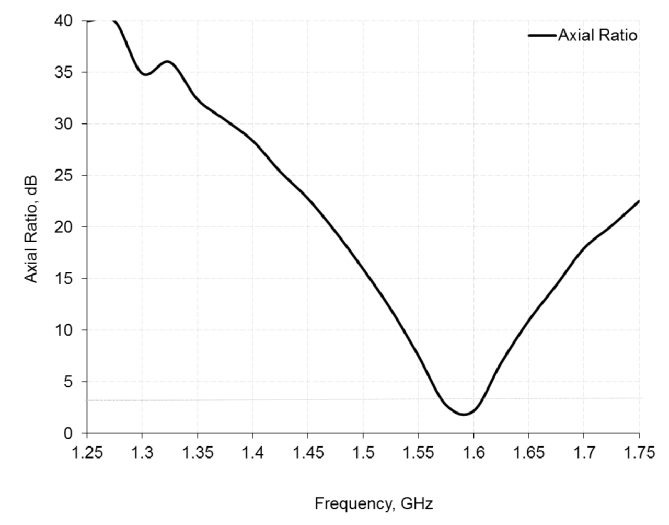

Fig. 1 Simulated axial ratio

A metallic patch of the dimensions in Fig. 1 and Table I was fabricated and is shown in Fig.5. 25 layers were necessary in order to achieve low resistance on the radiator. A very reasonable resistance of $0.4 \mathrm{ohms}$ was measured across the patch after the process was completed. The nature of the deposition of the silver, led to an uneven surface profile as shown in Fig.6. The surface roughness of the printed patch was measured to be $7183 \mathrm{~nm}$. 


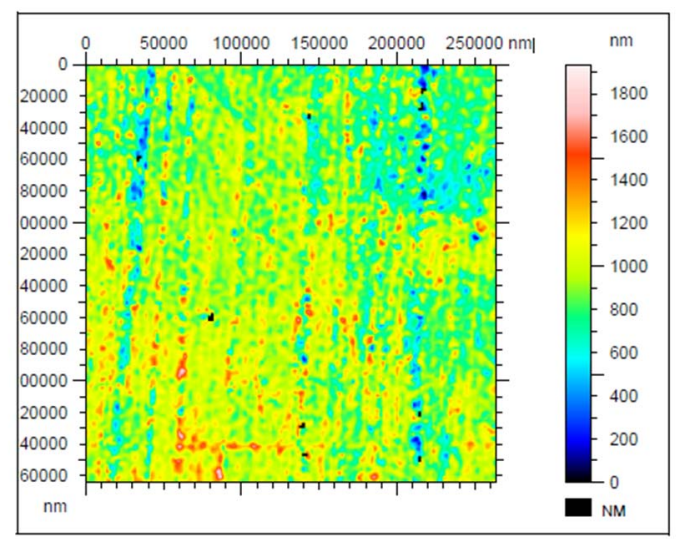

Fig.4 Surface profile for the 3D printed substrate

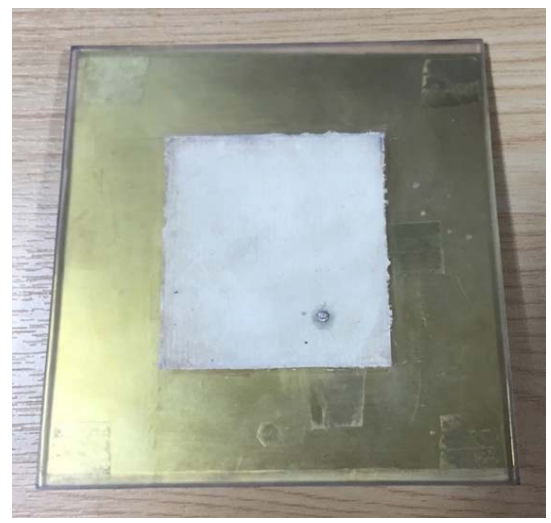

Fig.5 Inkjet printed antenna on SLA substrate

To complete the fabrication, a brass ground plane of $100 \mathrm{~mm}$ by $100 \mathrm{~mm}$ was placed on the underside of the substrate. A $1.3 \mathrm{~mm}$ hole was then drilled through the ground plane and the substrate at the location indicated in Fig. 1 to place the SMA connector for the feed line. The SMA connecter was soldered to the ground plane. Silver epoxy glue was used to connect the patch as soldering could damage the substrate and antenna.

\section{B. Measurements}

The measured S11 response of the patch antenna is shown in Fig. 7. Measurements compared very well with simulations. The resonant frequency was measured at $1.6 \mathrm{GHz}$ with a reflection coefficient of $-15 \mathrm{~dB}$ and $\mathrm{a}-10 \mathrm{~dB}$ bandwidth of $7 \%$. There was a second resonant mode at about $2.4 \mathrm{Gz}$. The resonant frequencies were less than $1 \%$ higher than those obtained in the simulations.

The simulated radiation patterns are shown in Fig. 8. Patterns were those expected for a patch antennas. The directivity was $7 \mathrm{~dB}$ at $1.6 \mathrm{GHz}$ and $5 \mathrm{~dB}$ at $2.4 \mathrm{GHz}$.

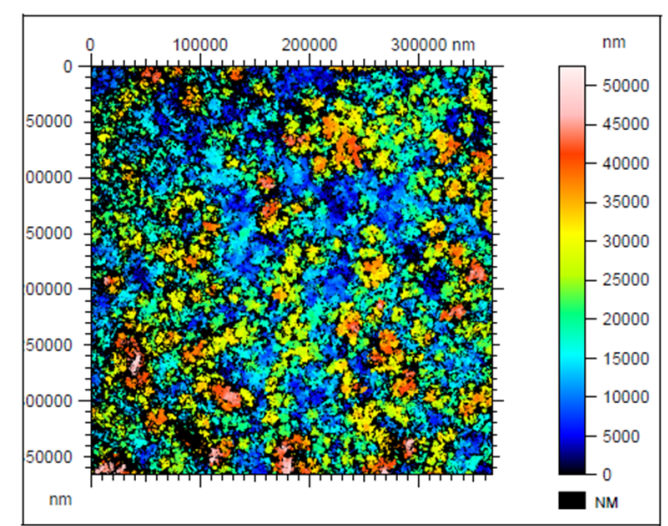

Fig.6 Surface profile for the 3D printed substrate with the layers of conductive silver ink

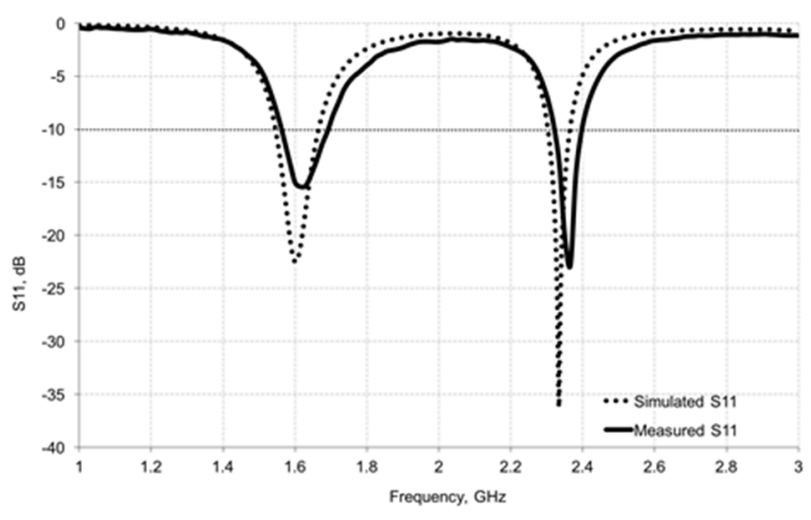

Fig.7 Measured and simulated $\mathrm{S}_{11}$

\section{CONCLUSIONS AND DISCUSSIONS}

The combination of stereolithography (SLA) and inkjet printing has been proven to be suitable for the fabrication of antennas. This can be realized using the low cost printing equipment described in this paper. SLA printing produces surfaces which are sufficiently smooth to support inkjet printing. An inkjet printing technique employing silver ink and ascorbic acid seems to adhere very well to the surface of the SLA substrate. It also produces relatively low resistance when a large number of layers are printed.

A patch antenna was designed, fabricated and tested in this demonstration. The measured results of the printed antenna compared very well with the simulations. Only a small change in the resonant frequencies was found. The performance is comparable to previously reported work, but with the advantage of using lower cost printing machines which are suitable for home use. Although the design is relatively simple, it opens up opportunities in terms of antennas printed on modified substrates. These altered substrates may have internal features which can improve the performance of the antenna [4]. Furthermore, there is a range of SLA resin with different properties which can lead to antennas customized to a specific application. Another further possibility is to control the 
deposition of the metallic layers, and therefore optimize the currents in the antennas as well as the amount of silver ink used.

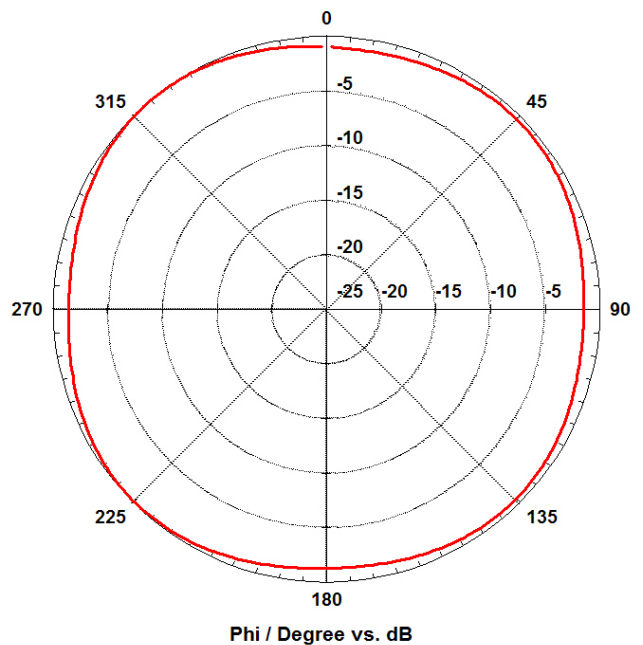

(a) x-y plane

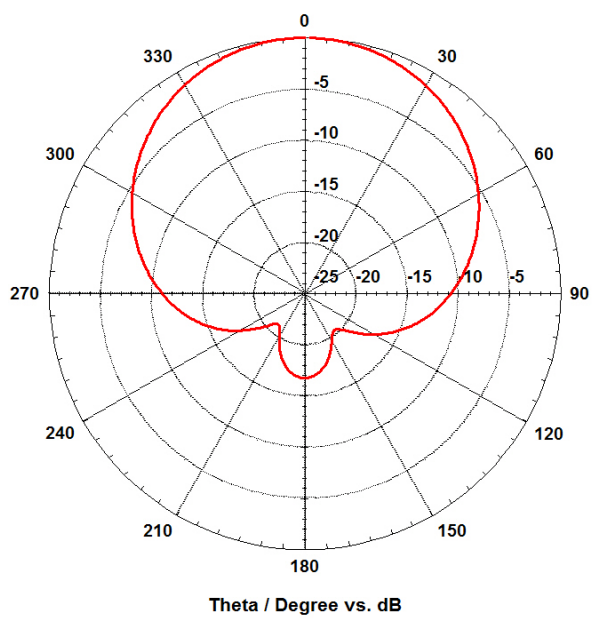

(b) x-z plane

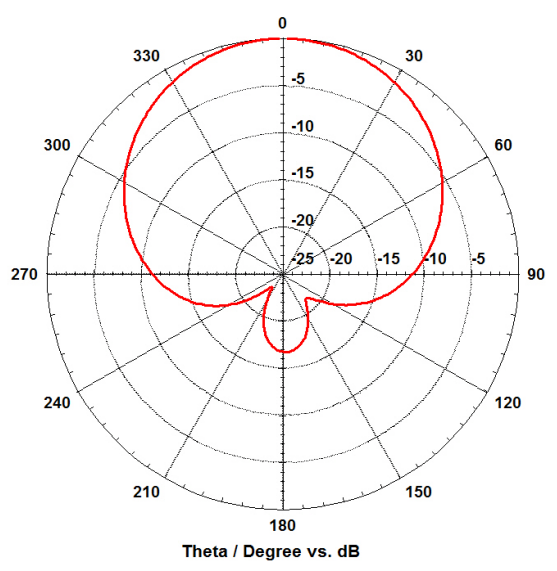

(c) y-z plane

Fig.8 Computed radiation patterns

\section{ACKNOWLEDGMENT}

The author would like to thank Simon Jakes for the fabrication of the antennas. This work was funded by a grant from the UK Royal Society, and by a UK EPSRC High Value Manufacturing Fellowship.

\section{REFERENCES}

[1] K. Wang, Y. Zhao, Y. Chang, Z. Qian, C. Zhang, B. Wang, M. Vannan and M. Wang, "Controlling the mechanical behavior of dual-material 3D printed meta-materials for patient-specific tissue-mimicking phantoms", Materials \& Design, vol. 90, pp. 704-712, 2016.

[2] E. Macdonald, R. Salas, D. Espalin, M. Perez, E. Aguilera, D. Muse and R. Wicker, "3D Printing for the Rapid Prototyping of Structural Electronics", IEEE Access, vol. 2, pp. 234-242, 2014.

[3] M. D'Auria, W. Otter, J. Hazell, B. Gillatt, C. Long-Collins, N. Ridler and S. Lucyszyn, "3-D Printed Metal-Pipe Rectangular Waveguides", IEEE Transactions on Components, Packaging and Manufacturing Technology, vol. 5, no. 9, pp. 1339-1349, 2015.

[4] J. Tribe, J. Vardaxoglou, W. Whittow and R. Kay, "Additively manufactured heterogeneous substrates for three-dimensional control of local permittivity", Electronics Letters, vol. 50, no. 10, pp. 745-746, 2014.

[5] B. Sanz-Izquierdo and E. A. Parker, "3-D Printing of Elements in Frequency Selective Arrays," IEEE Trans. Antennas Propagat., vol. 62, pp. 6060-6066, Dec 2014.

[6] B. Sanz-Izquierdo and E. Parker, "Frequency selective surfaces formed by partially metalising 3D printed shapes", 2015 9th European Conference on Antennas and Propagation (EuCAP), pp. 1-4, 2015.

[7] S. Jun, B. Sanz-Izquierdo and E. A. Parker, "3D printing technique for the development of non-planar electromagnetic bandgap structures for antenna applications," Electron. Lett., vol. 52, no. 3, pp. 175-176, Feb. 2016.

[8] B. Sanz-Izquierdo and S. Jun, "WLAN antenna on 3D printed bracelet and wrist phantom", 2014 Loughborough Antennas and Propagation Conference (LAPC), pp. 372 - 375, 2014.

[9] S. Jun, B. Sanz-Izquierdo and M. Summerfield, "UWB antenna on 3D printed flexible substrate and foot phantom", 2015 Loughborough Antennas \& Propagation Conference (LAPC), pp. 1-5, 2015.

[10] S. Khan, L. Lorenzelli and R. Dahiya, "Technologies for Printing Sensors and Electronics Over Large Flexible Substrates: A Review", IEEE Sensors J., vol. 15, no. 6, pp. 3164-3185, 2015.

[11] Y. Kawahara et al., "Instant Inkjet Circuits: Lab-Based Inkjet Printing to Support Rapid Prototyping of UbiComp Devices," Proc. 2013 ACM Int'l Joint Conf. Ubiquitous Computing (UbiComp 13), pp. 363-372, 2013.

[12] R. Vyas et al., "Paper-based RFID-enabled wireless platforms for sensing applications," IEEE Trans. Microw. Theory Tech., vol. 57, no. 5, pp. 1370-1382, May 2009.

[13] S. Kim, R. Vyas, A. Georgiadis, A. Collado, M. M. Tentzeris, "Inkjetprinted RF energy harvesting and wireless power trasmission devices on paper substrate," Digest of EuMC, pp. 983-986, Oct. 2013.

[14] G. Shaker, S. Safavi-Naeini, N. Sangary and M. Tentzeris, "Inkjet Printing of Ultrawideband (UWB) Antennas on Paper-Based Substrates", IEEE Antennas Wirel. Propag. Lett., vol. 10, pp. 111-114, 2011.

[15] J. C. Batchelor, E. A. Parker, J. A. Miller, V. Sanchez-Romaguera, and S. G. Yeates, "Ink jet printing of frequency selective surfaces," Electron. Lett., vol. 45, no. 1, pp. 7-8, Jan. 2009.

[16] J. Kimionis, M. Isakov, B. Koh, A. Georgiadis and M. Tentzeris, "3DPrinted Origami Packaging With Inkjet-Printed Antennas for RF Harvesting Sensors", IEEE Trans. Microw. Theory Tech., vol. 63, no. 12, pp. 4521-4532, 2015.

[17] Formlabs ( http://formlabs.com/ ), accesed 10/06/2016

[18] Cartesian Co (http://cartesianco.com/ ), accessed 10/06/2016

[19] S. M. Bidoki, D. M. Lewis , M. Clark, A. Vakorov , P. A. Miller and D. McGorman, "Ink-jet fabrication of electronic components", $J$. Micromech. Microeng., vol. 17, no. 5, pp. 967-974, 2007. 